\title{
Evaluation of Specific Risk Factors and Outcomes of Colistin-Resistant Klebsiella pneumoniae Infections in a Tertiary Care Centre - An Observational Study
}

\author{
Prakash Shastri* and Shamanth A Shankarnarayan ${ }^{2}$ \\ ${ }^{1}$ Department of Critical Care \& Emergency Medicine, Sir Ganga Ram Hospital, India \\ ${ }^{2}$ Department of Medical Microbiology, Postgraduate Institute of Medical Education and Research, India
}

\begin{abstract}
Background: Incidence of multidrug resistant Klebsiella pnumoniae infections are increasing globally especially in ICUs.

Aim: We evaluated the burden of colistin resistant K. pneumoniae (ColR KP) and the risk factors associated with the outcome of these patients.

Methods: Consecutive patients developing K. pneumoniae infections were included. K. pneumoniae from endotracheal tube and catheterized urine sample, having cell count $<10^{5} \mathrm{cfu} / \mathrm{ml}$, and which did not necessitate a change in antibiotics as per the treating physicians was considered as colonizer. Demographic and clinical details were collected and samples were processed as per standard protocol. Any growth was identified and its antimicrobial susceptibility was carried out by using Vitek 2 automated system. Minimum inhibitory concentration of $>4 \mu \mathrm{g} / \mathrm{ml}$ for Colistin was considered as resistant. The resistant isolates were confirmed with Broth microdilution method. Risk factor associated with the outcome of ColR KP was analyzed.
\end{abstract}

\section{OPEN ACCESS}

*Correspondence:

Prakash Shastri, Department of Critical Care \& Emergency Medicine, Sir Ganga Ram Hospital, Rajinder Nagar, New Delhi-110060, India, Tel: +91 9810937295;

E-mail: prakashshastri@live.in

Received Date: 12 Jul 2021

Accepted Date: 30 Aug 2021

Published Date: 08 Sep 2021

Citation:

Shastri P, Shankarnarayan SA. Evaluation of Specific Risk Factors and Outcomes of Colistin-Resistant Klebsiella pneumoniae Infections in a Tertiary Care Centre - An Observational Study. Am J Clin Microbiol Antimicrob. 2021; 4(2): 1054

Copyright $(2021$ Prakash Shastri. This is an open access article distributed under the Creative Commons Attribution License, which permits unrestricted use, distribution,

and reproduction in any medium, provided the original work is properly cited.
Findings: Burden of K. pneumoniae infection was 50.02 per 1000 admissions. K. pneumonie ( $\mathrm{n}=155)$ was isolated from patients with ventilator associated pneumonia $(84,54.2 \%)$, followed by blood stream infection $(49,31.6 \%)$ and urinary tract infection $(22,14.2 \%)$. ColR KP and intermediate (ColI $\mathrm{KP})$ isolates were $58(37.41 \%)$ and 97 (62.6\%) respectively. Among ColR KP infected patients 32 (55.1\%) died whereas 26 (44.8\%) patients were discharged. Higher mortality was witnessed in ColI $\mathrm{KP}$ cases $(75,77.3 \%)$ compared to ColR-KP cases $(32,55.1 \%)(\mathrm{p}=0.004 ; \mathrm{OR}=2.77 ; 95 \% \mathrm{CI}=1.37$ to 5.59). Colistin resistance and presence of central line were independently associated with mortality.

Conclusion: Colistin resistant K. pneumoniae infections among ICU patients are on rise. Presence of central venous catheter and resistance to colistin were independent predictors of mortality.

Keywords: Colistin Resistant Klebsiella; Carbapenem resistance; Risk factors; Multidrug resistance

\section{Abbreviations}

Colr KP: Colistin Resistant Klebsiella Pneumoniae; Coli KP: Colistin Intermediate Klebsiella Pneumoniae; ICU: Intensive Care Unit; KPC: Klebsiella Pneumoniae Carbapenamase; CFU: Colony Forming Unit

\section{Introduction}

The incidence of Carbapenem-resistant K. pneumoniae first reported in 1993 [1], has continued to increase globally, with such infections becoming a threat to ICU patients because of their high mortality rate and limited treatment options available [2,3]. This has led to increase use of tigecycline and colistin as the last resort treatment for infections caused by Multidrug-Resistant (MDR) $K$. pneumoniae.

Carbapenem resistance is mostly due to serine-based betalactamases which are Class A Carbapanemases $\beta$-lac first identified in the US in 1996 [4]. In Greece, plasmids and integrons encoding VIM-1 MBL have spread widely among K. pneumoniae, with $42 \%$ of bloodstream isolates now carrying these enzymes or K. Pneumoniae Carbapenamase (KPC) types [5]. In India Class B (Metallo-Beta-Lactamases, MBL) and Class D 9 (Oxacillinase) are enzymes responsible for 
contributing to multidrug resistance [6].

Colistin-resistant K. pneumoniae (ColR-KP) rate in ICUs across Europe and US is $8.2 \%$ and $2.7 \%$ respectively [7]. Few European countries such as Romania, Greece and Italy have reported higher incidences of $25.8 \%, 19.9 \%$ and $15.4 \%$ ColR-KP infections respectively [8]. In India there is a paucity of systematic data on ColRKP but corroborative findings suggest that these resistant strains are widely prevalent in Indian ICUs [9]. A case series from South Indian hospital reported ColR-KP infections in 33\% (6/18) cases, and gram negative bacterial isolates resistant to both carbapenems and Colistin in $30 \%(27 / 89)$ cases [10]. Therefore, the present study was planned to study the prevalence of ColR-KP in our ICU and to understand the potential risk factors that determine the outcome of patients infected with ColR-KP.

\section{Materials and Methods}

\section{Study design, setting, and patients}

Study design: This study is a prospective observational study.

Setting: The study was carried out in a 46 bedded multi-specialty Intensive Care Unit (ICU) in a tertiary care hospital which receives patients from a large part of North India as well as North Eastern states. Patients admitted in the ICU during a period of one year (April $1^{\text {st }}, 2017$ to March 31 $1^{\text {st }}, 2018$ ) from whom K. pneumoniae was isolated from either blood, respiratory or urine sample were included in the study. Bacterial count exceeding $>10^{5}$ Colony Forming Units $(\mathrm{CFU} / \mathrm{ml})$ in the endotracheal aspirate and catheterized urine sample with $>10$ pus cells $/ \mathrm{ml}$ were considered as pathogens. If the colony count was $<10^{5}(\mathrm{CFU}) / \mathrm{ml}$ and not accompanied by a change in antibiotics, the isolates were labeled as colonizers. Each patient was included in the study only once, at the time of the first isolation. A ColR-KP Blood Stream Infection (BSI) was defined as the presence of at least one ColR-KP - positive culture with concomitant signs of organ dysfunction. The case was defined as healthcare-associated or hospital-acquired infection as per standard definitions [11].

A case: control design was used to study risk factors for ColR-KP infection. The study was approved by the Institute Ethics committee and an informed consent was taken from the next of kin.

Data collection: A predesigned case record form was used to save the demographical (age and sex) and clinical data of each patient. The clinical data consisted of specialty (medical or surgical), date of admission, co-morbidity (diabetes mellitus, steroid intake, transplant), use of medical devices (ventilator, central venous catheter, urinary catheter), APACHE score, date of detecting first $K$. pneumoniae infection, type of K. pneumoniae infection (CatheterRelated Bloodstream Infection (CRBSI), Ventilator-Associated Pneumonia (VAP), Catheter-Associated Urinary Tract Infection (CAUTI).

The identification of the isolates and antimicrobial susceptibility testing was done by compact automated system (VITEK 2, BioMe rieux, France) and interpreted according to Clinical and Laboratory Standard Institute (CLSI) guidelines 2015. Colistin resistance was defined as Minimum Inhibitory Concentration (MIC) of $>4 \mu \mathrm{g} / \mathrm{ml}$ $[7,12]$. The resistant isolates were tested again by Broth microdilution. Antimicrobial therapy given and the final outcome of the patient (ICU discharge or death) were also recorded.

CRBSI, VAP and CAUTI were defined in accordance with the CDC/NHSN definitions [13]. APACHE II score was noted among these patients. In patients who were prescribed colistin, it was administered as a continuous infusion over a three-hour period which is the protocol in the ICU. Diagnostic and therapeutic management for all patients was not standardized and decisions were made at the discretion of the attending physician.

Data evaluation: All details from the case record forms were entered into Microsoft excel spreadsheet. The continuous variables were described as mean \pm SD in accordance with standard formulae. Categorical variables were expressed as percentages which includes susceptibility to colistin and final outcome. Student t-test or Mann Whitney-U test were applied to evaluate continuous variables. Chisquare test or Fisher's exact test was applied to test the association between categorical variables. Odds Ratio (OR) and 95\% Confidence Interval (CI) was calculated and a $\mathrm{p}$ value of $<0.05$ was considered as statistically significant. Variables with a $p$ value of $<0.05$ were taken up for multivariate analysis to identify independent predictors of clinical outcome. All the statistical analysis was carried out using Jamovi Software version 1.6.14.

Risk factors for acquiring ColR KP infection was assessed by comparing a total of 58 patients with ColR KP to two groups: Colistin-intermediate ColI-KP cases $(n=97$, control A) and patients classified as colonizers ( $\mathrm{n}=77$, control $\mathrm{B})$. The discrimination ability of the models was assessed by estimating the area under the receiver operating characteristic (ROC) curve. Calibration of the models was assessed using the Hosmer-Leme show test for goodness of fit.

\section{Results}

Demographical and clinical profile of the patients (Table 1).

A total of 3,099 patients were admitted to the ICU during the study period out of which 155 patients developed $K$. pneumoniae infection amounting to a burden of $50.02 \mathrm{~K}$. pneumoniae infections $/ 1000$ admissions. Median APACHE-II score at the time of admission was 19 (range 8-43). VAP was the most common ICU-acquired infection (84, 54.2\%), followed by CRBSI $(49,31.6 \%)$ and CAUTI in (22, $14.2 \%)$.

\section{Susceptibility profile of $K$. pneumoniae isolates}

Among $155 \mathrm{~K}$. pneumoniae infections, colistin resistant and intermediate isolates were $58(37.41 \%)$ and 97 (62.6\%) respectively. Remaining 77 (33.2\%) isolates were deemed as colonizers. Among these colonizers, $46(59.7 \%)$ were intermediate to colistin whereas remaining $31(40.3 \%)$ were resistant. Colistin was administered to $64 / 232(27.5 \%)$ patients initially as pre-emptive therapy. Later $34(14.65 \%)$ patients were additionally put on colistin after first antibiotic change. The decision to change antibiotics was either a clinical decision pending culture reports or based upon the culture sensitivity report. Thus a total of $98(42.2 \%)$ patients received colistin during their hospital stay. Among colistin resistant K. pneumoniae infected patients $32 / 58(55.1 \%)$ patients died whereas 26/58 (44.8\%) patients were alive to discharge. Only 17 patients yielding colistin resistant $K$. pneumoniae were administered with colistin as initial therapy. Later, in 12 of these 17 patients colistin was discontinued (except in 5 patients in whom colistin was continued at a higher dose as per the physician's discretion).

\section{Patient outcome and predictors of mortality}

The demographic details and other parameters of the entire cohort of 232 patients are given in Table 1. Demographical factors like age and male preponderance did not have any bearing on the final 
Table 1: Demographic and clinical characteristics of all the patients enrolled in the study.

\begin{tabular}{|c|c|}
\hline Characteristics & Observation $(n=232)$ \\
\hline \multicolumn{2}{|l|}{ Demographics } \\
\hline Age (years \pm SD) & $56.5 \pm 16.6$ \\
\hline Males (\%) & $164(70.7)$ \\
\hline \multicolumn{2}{|l|}{ Risk factors (\%) } \\
\hline Diabetes mellitus & $100(43.1)$ \\
\hline Steroid therapy & $40(17.2)$ \\
\hline Organ transplant & $15(6.5)$ \\
\hline \multicolumn{2}{|l|}{ Procedural interventions (\%) } \\
\hline Endotracheal intubation & $193(83.2)$ \\
\hline Central line insertion & $212(91.4)$ \\
\hline Urinary bladder catheterization & $224(96.6)$ \\
\hline \multicolumn{2}{|l|}{ Klebsiella Infections (\%) } \\
\hline CAUTI & $22(14.2)$ \\
\hline CRBSI & $49(31.6)$ \\
\hline VAP & $84(54.2)$ \\
\hline \multicolumn{2}{|l|}{ APACHE-II score } \\
\hline Median \pm SD & $19.4 \pm 5.6$ \\
\hline Range & 8 to 43 \\
\hline \multicolumn{2}{|l|}{ Duration of hospitalization (days) } \\
\hline Median (Range) & 21 (2 to 117 days) \\
\hline \multicolumn{2}{|c|}{ Colistin sensitivity of all isolates (\%) } \\
\hline Intermediate & $133(57.3)$ \\
\hline Resistant & $99(42.7)$ \\
\hline
\end{tabular}

SD: Standard Deviation; IQR: Interquartile Range; CAUTI: Catheter Associated Urinary Tract Infection; CRBSI: Catheter Related Blood Stream Infection; VAP: Ventilator Associated Pneumonia; APACHE: Acute Physiology and Chronic Health Evaluation

outcome and were comparable among survivors and non survivors. Overall, 107 (69.03\%) K. pneumoniae infected patients succumbed and $48(30.0 \%)$ were discharged from the ICU. Median length of hospital stay was 24 days for patients who survived and 20 days for patients who died $(\mathrm{p}=0.025)$.

To assess risk factors for ColR KP $(n=58)$ cases were compared to two control populations. The first control group (control $\mathrm{A}, \mathrm{n}=97$ ) had patients with K. pneumoniae infections which are intermediate to colistin. Whereas, the second control group harbored K. pneumoniae as colonizers (control group $\mathrm{B}, \mathrm{n}=77$ ). Table $2 \& 3$ depicts results of univariate analysis. In the first comparison among ColR-KP and ColI-KP revealed higher mortality in ColI KP cases $(75,77.3 \%)$ compared to ColR-KP cases $(32,55.1 \%)(\mathrm{p}=0.004$; OR=2.77; $95 \%$ $\mathrm{CI}=1.37-5.59$ ). Among patients with diabetes mellitus the proportion was higher of ColR-KP (50.0\%) compared to ColI-KP (34.0\%). In the second comparison, ColR-KP verses colonizers, no significant difference was witnessed between ColR-KP and colonizers, but risk factors like transplant patients $(\mathrm{p}=0.029 ; \mathrm{OR}=0.194 ; 95 \% \mathrm{CI}=0.038$ to 0.97 ), patients on steroids ( $\mathrm{p}=0.029 ; \mathrm{OR}=0.029 ; 95 \% \mathrm{CI}=0.114$ to 0.924 ) were significantly associated with ColR-KP infection. In the multivariate analysis, Colistin resistance and presence of central line were independently associated with mortality (Table 4).

\section{Discussion}

Studies evaluating the specific risk factors and clinical outcomes of patients infected with colistin-resistant K. pneumoniae in Indian ICU are scarce $[12,14]$. Globally, there are only a handful of studies addressing similar concern. In this study, we report $38.3 \%$ of patients were infected and colonized with colistin resistant $K$. pneumoniae among ICU patients (25\% and $13.3 \%$ respectively). In a previous study, active surveillance in an ICU revealed that $24.4 \%$ were colonized in the gastrointestinal tract by colistin-resistant KPC-producing $K$. pneumoniae [15]. In contrast, we found $40.2 \%$ of our patients were colonized with ColR-KP. This high colonization rate may explain the higher incidence of ColR-KP infection observed in the present study. Here we report only $37.4 \%$ of the K. pneumoniae infections which were resistant to Colistin. This is similar to studies from Greece [16],

Table 2: Comparison of various parameters of patients infected with colistin resistant and colistin intermediate Klebsiella pneumoniae.

\begin{tabular}{|c|c|c|c|c|}
\hline Variables & Colistin resistant $(n=58)$ & Colistin intermediate $(n=97)$ & $P$ value & Odds ratio \\
\hline Age (Mean \pm SD) & $58.6 \pm 17.3$ & $55.1 \pm 16.4$ & 0.203 & \\
\hline Male & $45(77.5 \%)$ & $69(71.2 \%)$ & \multirow{2}{*}{0.378} & \multirow{2}{*}{1.40 (0.695 to 3.00$)$} \\
\hline Female & $13(22.5) \%$ & $28(28.8 \%)$ & & \\
\hline APACHE (Mean \pm SD) & $18.5 \pm 4.36$ & $20 \pm 6.02$ & 0.099 & \\
\hline Total hospital days (Mean \pm SD) & $31.9 \pm 29.2$ & $31.2 \pm 30.3$ & 0.873 & \\
\hline \multicolumn{5}{|l|}{ Speciality } \\
\hline Medical & $41(70.6 \%)$ & $62(63.9 \%)$ & \multirow{2}{*}{0.38} & \multirow{2}{*}{1.36 (0.675 to 2.74$)$} \\
\hline Surgical & $17(29.4 \%)$ & $35(36.1 \%)$ & & \\
\hline \multicolumn{5}{|l|}{ Risk Factors } \\
\hline Transplant & $7(12.0 \%)$ & $6(6.1 \%)$ & 0.201 & 2.08 (0.664 to 6.53$)$ \\
\hline Diabetes Mellitus & $29(50.0 \%)$ & $33(34.0 \%)$ & $0.049^{*}$ & 1.94 (0.998 to 3.77$)$ \\
\hline Steroids therapy & $12(20.6 \%)$ & $22(22.6 \%)$ & 0.772 & 0.889 (0.402 to 1.97$)$ \\
\hline Endotracheal Intubation & $44(75.8 \%)$ & $86(88.6 \%)$ & $0.036^{\star}$ & 0.402 (0.169 to 0.959$)$ \\
\hline Central line & $48(82.7 \%)$ & $92(94.8 \%)$ & $0.014^{*}$ & 0.261 (0.084 to 0.807$)$ \\
\hline Urinary catheter & $54(93.1 \%)$ & $96(98.9 \%)$ & $0.045^{*}$ & 0.141 (0.015 to 1.29$)$ \\
\hline Blood culture positivity & $19(32.7 \%)$ & $30(30.9 \%)$ & 0.879 & 1.06 (0.525 to 2.12$)$ \\
\hline Respiratory culture positivity & $31(53.4 \%)$ & $53(54.6 \%)$ & 0.778 & $0.91(0.472$ to 1.75$)$ \\
\hline Urine culture positivity & $8(13.7 \%)$ & $14(14.4 \%)$ & 0.801 & 0.886 (0.346 to 2.27$)$ \\
\hline \multicolumn{5}{|l|}{ Outcome } \\
\hline Survivors & $26(44.8 \%)$ & $22(22.6 \%)$ & \multirow{2}{*}{$0.004^{*}$} & \multirow{2}{*}{2.77 (1.37 to 5.59$)$} \\
\hline Non Survivors & $32(55.1 \%)$ & $75(77.3 \%)$ & & \\
\hline
\end{tabular}


Table 3: Comparison of various risk factors among patients with ColR-KP and colonizers.

\begin{tabular}{|c|c|c|c|c|}
\hline & Colistin resistant KP $(n=58)$ & Colonizer KP (n=77) & $P$ value & Odds ratio $(95 \% \mathrm{Cl})$ \\
\hline Age (Mean \pm SD) & $58.6 \pm 17.3$ & $56.6 \pm 16.4$ & 0.501 & \\
\hline Male & $45(77.5 \%)$ & $50(64.9 \%)$ & \multirow{2}{*}{0.111} & \multirow{2}{*}{0.535 (0.247 to 1.16$)$} \\
\hline Female & $13(22.5) \%$ & $27(35.1 \%)$ & & \\
\hline APACHE (Mean \pm SD) & $18.5 \pm 4.36$ & $19.4 \pm 5.78$ & 0.327 & \\
\hline Total hospital days (Mean \pm SD) & $31.9 \pm 29.2$ & $25.3 \pm 18.1$ & 0.107 & \\
\hline \multicolumn{5}{|l|}{ Speciality } \\
\hline Medical & $41(70.6 \%)$ & $57(74.0 \%)$ & \multirow{2}{*}{0.667} & \multirow{2}{*}{1.18 (0.552 to 2.53$)$} \\
\hline Surgical & $17(29.4 \%)$ & $20(26.0 \%)$ & & \\
\hline Transplant & $7(12.0 \%)$ & $2(2.6 \%)$ & $0.029^{*}$ & 0.194 (0.038to 0.97) \\
\hline Diabetes & $29(50.0 \%)$ & $38(49.4 \%)$ & 0.94 & 0.974 (0.493 to 1.93$)$ \\
\hline Steroids & $12(20.6 \%)$ & $6(7.8 \%)$ & $0.029^{*}$ & $0.029(0.114$ to 0.924$)$ \\
\hline ET tube & $44(75.8 \%)$ & $57(74.0 \%)$ & 0.808 & 0.907 (0.412 to 1.99$)$ \\
\hline Central line & $48(82.7 \%)$ & $72(93.5 \%)$ & $0.049^{*}$ & $3.0(0.965$ to 9.32$)$ \\
\hline Urinary catheter & $54(93.1 \%)$ & $74(96.1 \%)$ & 0.436 & 1.83 (0.393 to 8.50$)$ \\
\hline \multicolumn{5}{|l|}{ Outcome } \\
\hline Discharged & $26(44.8 \%)$ & $31(40.3 \%)$ & \multirow{2}{*}{0.595} & \multirow{2}{*}{$0.829(0.416$ to 1.65$)$} \\
\hline Expired & $32(55.1 \%)$ & $46(59.7 \%)$ & & \\
\hline
\end{tabular}

*Statistically significant

Table 4: Multivariate analysis to evaluate the outcome among resistant and sensitive group Model =AUC: $73.2 \%$, Accuracy $=77.4 \%, \mathrm{R}^{2}=0.162, \mathrm{p}=0.021$.

\begin{tabular}{|l|c|c|}
\hline \multicolumn{1}{|c|}{ Risk factors } & OR $(\mathbf{9 5 \%} \mathbf{~ C l )}$ & P Value \\
\hline Central line & $0.043(0.006-0.278)$ & $<0.001$ \\
\hline Resistant to colistin & $2.34(1.065-5.16)$ & 0.034 \\
\hline
\end{tabular}

and Italy [12]. Where they have reported the incidence of ColR-KP infection at $46.4 \%$ and $33.3 \%$ respectively. However, contemporary Indian studies have reported colistin resistance in Klebsiella ranging from $4 \%$ [17], to $5.6 \%$ [8], but the relative number of cases was very less.

Unlike previous study, high mortality was witnessed among patients infected with ColI-KP compared to ColR-KP (77.3\% against 55.1\%) [12]. In literature, studies have reported higher mortality among patients infected with ColR-KP and colistin resistance has been shown to be an independent predictor of mortality $[8,12,18]$. Maybe the increased use of this drug in recent years, especially as monotherapy, could be the reason for increased mortality. Again resistance to colistin could be simply a marker of the patient's severity as shown in infections caused by other resistant microorganisms such as Pseudomonas aeruginosa [19]. However, physicians should be aware of such an occurrence for its implications on treatment and outcome.

In our study, majority (58.6\%) of the patients infected with ColRKP did not receive colistin. Intra ICU transmission of ColR-KP to susceptible patients cannot be ruled out. However, these results indicate that even though colistin administration plays an important role in the development of resistance in K. pneumoniae it may not be the only risk factor. Health care workers may also play a pivotal role in transmission of resistant isolates to non-colonized patients [20].

The 30-day mortality among patients with colistin-resistant KP was $55.1 \%$ which is considerably higher compared to recent studies which have reported rates ranging from $30.8 \%$ to $51 \%$ [12,21]. A
Taiwan study on the other hand reported a mortality of 71\% [22]. Again, the mortality among ColR-KP infections was significantly lower (55.1.9\%) than ColI-KP infections $(77.3 \%)$ in our ICU. However, the mortality rate among colistin sensitive K. pneumoniae infected patients is lower (39.4\%) in a study from Italy [12].

Among the demographical factors, age of the patient did not have any bearing on the overall outcome in the present study. This was in accordance with prior studies wherein age of the patient did not vary with colistin susceptibility or overall outcome [16,23]. Ritcher et al. [23] however, reported higher age to be significantly associated with ColR-KP infections as compared to ColI-KP, though they have not compared it with the final clinical outcome of the patients. This age-related difference in outcome among ColR-KP as compared to Carbapenem-Resistant KP could be an interesting topic of further research. A $70.7 \%$ male preponderance was noticed in the present study but male gender did not predispose to poor outcome. Male preponderance has been suggested by nearly all prior studies but no statistically significant gender predisposition could be attributed to the clinical outcome $[10,16,23-25]$.

The mortality from K. pneumoniae infection in our ICU was $69 \%$. This was higher (43.4\%) compared to a multi-centric Italian ICU study [26]. The crude mortality associated with carbapenem-resistant $K$. pneumoniae infection ranges from $20 \%$ to $70 \%$, being as high as $45 \%$ following bloodstream infection and even higher when secondary to pulmonary source [27-29]. The prevalence of $81 \%$ carbapenemresistant isolates and 54.2\% VAP infections could have contributed to higher mortality in our ICU. While Giacobbe et al. [12], have reported higher mortality in ColR-KP (51.4\%) compared to ColI-KP (39.4\%) $(\mathrm{p}=0.02)$ infections. Zarkotou et al. [28] have shown no significant difference in survival among the two groups $(\mathrm{p}=0.53)$. Interestingly, the mortality incidence among ColR-KP infections was significantly lower $(55.1 .9 \%)$ than ColI-KP infections (77.3\%) in our ICU. These observations lead us to ponder over the following points: Firstly, several researchers have noticed that colistin administration was 
associated with the emergence of ColR-KP $[10,16,28]$, underscoring the absolute need of avoiding unnecessary colistin use in clinical practice. It is possible that our patients who were administered colistin based on the initial susceptibility report progressed to eventually develop ColR-KP strains. Secondly, the reduced survival in ColRKP infection could be attributable to inadequate empiric treatment options left for such strains. This signifies the horizontal transfer of ColR-KP of colonized patient to another non-colonized patient via medical and nursing personnel [16]. Hence, strict adherence to infection prevention and control practices, especially the component of hand hygiene, should be emphasized in ICU.

The study has certain limitations such as unmeasured factors like previous colistin therapy, its duration, dosage and regimen (whether monotherapy or combination) could have contributed to the emergence of ColR-KP in our ICU. No molecular analysis was carried out to rule out inter-patient spread by colistin resistant strains within ICU, as it was beyond the scope of this study. Further, we did not evaluate the differences in mortality, if any, in different drug combination schemes were being used (colistin monotherapy vs. colistin + carbapeneem vs. colistin + aminoglycoside, etc). Few earlier studies $[16,28]$ have already addressed these issues and there are still an unresolved debate pertain over benefits of monotherapy $v s$. combination therapy with Colistin [30]. Another possible limitation is that colistin susceptibility was performed by automated system (Vitek) and by broth microdilution technique only for confirmation in the resistant cases. However, recent study indicated lower error rate with automated system (vitek) compared to other techniques used for colistin susceptibility where the results of Vitek were comparable to broth microdilution using glass-coated plates [31].

\section{Conclusion}

Colistin resistant $K$. pneumoniae infections as well as colonization among ICU patients are on rise. Diabetic patients were more frequently infected with colistin resistant $K$. pneumoniae. Patients with extraneous devices were more consistently infected with colistin intermediate K. pneumoniae. Presence of central venous catheter and resistance to colistin were independent predictors of mortality. Hence, with a very high rate of ColR-KP isolates in Indian ICUs, there is an urgent need to identify potential risk factors predisposing to infections due to MDR K. pneumoniae, and implementation of infection control practices. In the absence of new antimicrobial agents for the treatment of healthcare associated infections due to MDR strains, the implementation of proper prevention strategies and adequate staffing is essential to control their spread. Applying routine use of molecular analyses help in control of outbreaks as well as accurate detection of mutations conferring resistance.

\section{References}

1. Naas T, Nordmann P. Analysis of a carbapenem-hydrolyzing class A beta-lactamase from Enterobacter cloacae and of its LysR-type regulatory protein. Proc Natl Acad Sci USA. 1994;91(16):7693-7.

2. van Duin D, Kaye KS, Neuner EA, Bonomo RA. Carbapenem-resistant Enterobacteriaceae: A review of treatment and outcomes. Diagn Microbiol Infect Dis. 2013;75(2):115-20.

3. Gupta N, Limbago BM, Patel JB, Kallen AJ. Carbapenem-Resistant Enterobacteriaceae: Epidemiology and prevention. Clin Infect Dis. 2011;53(1):60-7.

4. Livermore DM. Has the era of untreatable infections arrived? J Antimicrob Chemother. 2009;64(Suppl 1):i29-36.
5. Vatopoulos A. High rates of metallo-beta-lactamase-producing Klebsiella pneumoniae in Greece - a review of the current evidence. Euro Surveill. 2008;13(4):8023.

6. Castanheira M, Deshpande LM, Mathai D, Bell JM, Jones RN, Mendes RE. Early dissemination of NDM-1- and OXA-181-Producing Enterobacteriaceae in Indian Hospitals: Report from the SENTRY Antimicrobial Surveillance Program, 2006-2007. Antimicrob Agents Chemother. 2011;55(3):1274-8.

7. Munoz-Price LS, Poirel L, Bonomo RA, Schwaber MJ, Daikos GL, Cormican $\mathrm{M}$, et al. Clinical epidemiology of the global expansion of Klebsiella pneumoniae carbapenemases. Lancet Infect Dis. 2013;13(9):78596.

8. Capone A, Giannella M, Fortini D, Giordano A, Meledandri M, Ballardini $\mathrm{M}$, et al. High rate of colistin resistance among patients with carbapenemresistant Klebsiella pneumoniae infection accounts for an excess of mortality. Clin Microbiol Infect. 2013;19(1):E23-30.

9. Sodhi K, Mittal V, Arya M, Kumar M, Phillips A, Kajla B. Pattern of colistin resistance in Klebsiella isolates in an intensive care unit of a tertiary care hospital in India. J Infect Public. 2020;13:1018-21.

10. Manohar P, Shanthini T, Ayyanar R, Bozdogan B, Wilson A, Tamhankar AJ, et al. The distribution of carbapenem- And colistin-resistance in Gramnegative bacteria from the Tamil Nadu region in India. J Med Microbiol. 2017;66(7):874-83.

11. Garner JS, Jarvis WR, Emori TG, Horan TC, Hughes JM. CDC definitions for nosocomial infections. Am J Infect Control. 1988;16(3):128-40.

12. Giacobbe DR, Del Bono V, Trecarichi EM, De Rosa FG, Giannella M, Bassetti $\mathrm{M}$, et al. Risk factors for bloodstream infections due to colistinresistant KPC-producing Klebsiella pneumoniae: Results from a multicenter case-control-control study. Clin Microbiol Infect. 2015;21(12):1106.e1-8.

13. Horan TC, Andrus M, Dudeck MA. CDC/NHSN surveillance definition of health care-associated infection and criteria for specific types of infections in the acute care setting. Am J Infect Control. 2008;36(5):309-32.

14. Goel G, Hmar L, Sarkar De M, Bhattacharya S, Chandy M. ColistinResistant Klebsiella pneumoniae: Report of a cluster of 24 cases from a New Oncology Center in Eastern India. Infect Control Hosp Epidemiol. 2014;35(8):1076-7.

15. Papadimitriou-Olivgeris M, Bartzavali C, Christofidou M, Bereksi N, Hey J, Zambardi G, et al. Performance of Chromid $^{\circledR}$ CARBA medium for carbapenemases-producing Enterobacteriaceae detection during rectal screening. Eur J Clin Microbiol Infect Dis. 2014;33(1):35-40.

16. Papadimitriou-Olivgeris M, Bartzavali C, Spyropoulou A, Lambropoulou A, Sioulas N, Vamvakopoulou S, et al. Molecular epidemiology and risk factors for colistin- or tigecycline-resistant carbapenemase-producing Klebsiella pneumoniae bloodstream infection in critically ill patients during a 7-year period. Diagn Microbiol Infect Dis. 2018;92(3):235-40.

17. Mathur P, Khurana S, de Man TJB, Rastogi N, Katoch O, Veeraraghavan $\mathrm{B}$, et al. Multiple importations and transmission of colistin-resistant Klebsiella pneumoniae in a hospital in northern India. Diagn Microbiol Infect Dis. 2018;92(3):235-40.

18. Tumbarello M, Trecarichi EM, De Rosa FG, Giannella M, Giacobbe $\mathrm{DR}$, Bassetti M, et al. Infections caused by KPC-producing Klebsiella pneumoniae: Differences in therapy and mortality in a multicentre study. J Antimicrob Chemother. 2015;70(7):2133-43.

19. Peña C, Suarez C, Gozalo M, Murillas J, Almirante B, Pomar V, et al. Prospective multicenter study of the impact of Carbapenem resistance on mortality in Pseudomonas aeruginosa bloodstream infections. Antimicrob Agents Chemother. 2012;56(3):1265-72.

20. Papadimitriou-Olivgeris M, Christofidou M, Fligou F, Bartzavali C, Vrettos $\mathrm{T}$, Filos KS, et al. The role of colonization pressure in the dissemination of colistin or tigecycline resistant KPC-producing Klebsiella pneumoniae in 
critically ill patients. Infection. 2014;42(5):883-90.

21. Machuca I, Gutiérrez-Gutiérrez B, Gracia-Ahufinger I, Rivera Espinar F, Cano Á, Guzmán-Puche J, et al. Mortality associated with bacteremia due to Colistin-Resistant Klebsiella pneumoniae with high-level Meropenem resistance: Importance of combination therapy without Colistin and Carbapenems. Antimicrob Agents Chemother. 2017;61(8):e00406-17.

22. Menekşe Ş, Çağ Y, Işık ME, Şahin S, Hacıseyitoğlu D, Can F, et al. The effect of colistin resistance and other predictors on fatality among patients with bloodstream infections due to Klebsiella pneumoniae in an OXA-48 dominant region. Int J Infect Dis. 2019;86:208-11.

23. Richter SE, Miller L, Uslan DZ, Bell D, Watson K, Humphries R, et al Risk factors for Colistin Resistance among Gram-Negative Rods and Klebsiella pneumoniae isolates. Ledeboer NA, editor. J Clin Microbiol. 2018;56(9):e00149-18.

24. Chen C-Y, Tien F-M, Sheng W-H, Huang S-Y, Yao M, Tang J-L, et al Clinical and microbiological characteristics of bloodstream infections among patients with haematological malignancies with and without neutropenia at a medical centre in northern Taiwan, 2008-2013. Int J Antimicrob Agents. 2017;49(3):272-81.

25. Mouloudi E, Massa E, Papadopoulos S, Iosifidis E, Roilides I, Theodoridou $\mathrm{T}$, et al. Bloodstream infections caused by Carbapenemase-producing Klebsiella pneumoniae among intensive care unit patients after orthotopic liver transplantation: Risk factors for infection and impact of resistance on outcomes. Transplant Proc. 2014;46(9):3216-8.

26. Friedman ND. Health Care-Associated Bloodstream Infections in Adults: A reason to change the accepted definition of community-acquired infections. Ann Intern Med. 2002;137(10):791-7.
27. Sader HS, Farrell DJ, Flamm RK, Jones RN. Antimicrobial susceptibility of Gram-negative organisms isolated from patients hospitalised with pneumonia in US and European hospitals: Results from the SENTRY Antimicrobial Surveillance Program. Int J Antimicrob Agents. 2014;43(4):328-34.

28. Zarkotou O, Pournaras S, Tselioti P, Dragoumanos V, Pitiriga V, Ranellou $\mathrm{K}$, et al. Predictors of mortality in patients with bloodstream infections caused by KPC-producing Klebsiella pneumoniae and impact of appropriate antimicrobial treatment. Clin Microbiol Infect. 2011;17(12):1798-803.

29. Tumbarello M, Viale P, Viscoli C, Trecarichi EM, Tumietto F, Marchese A, et al. Predictors of mortality in bloodstream infections caused by Klebsiella pneumoniae Carbapenemase-producing K. pneumoniae: Importance of combination therapy. Clin Microbiol Infect. 2012;55(7):943-50.

30. Vardakas KZ, Mavroudis $\mathrm{AD}$, Georgiou M, Falagas ME. Intravenous colistin combination antimicrobial treatment vs. monotherapy: A systematic review and meta-analysis. Int J Antimicrob Agents. 2018;51(4):535-47.

31. Singhal L, Sharma M, Verma S, Kaur R, Britto XB, Kumar SM, et al. Comparative evaluation of broth microdilution with polystyrene and glass-coated plates, agar dilution, E-test, Vitek, and disk diffusion for susceptibility testing of colistin and Polymyxin B on CarbapenemResistant clinical isolates of Acinetobacter baumnnii. Microb Drug Resist. 2018;24(8):1082-8. 